\title{
Nano-Yarn Carbon Nanotube Fiber Based Enzymatic Glucose Biosensor
}

Zhigang Zhu ${ }^{\mathrm{a}} \uparrow$, Wenhui Song ${ }^{\mathrm{b}^{*}}$, Krishna Burugapalli $^{\mathrm{a}}$, Francis Moussy ${ }^{\mathrm{a}}$, Yali Li $^{\mathrm{c}}$, Xiahua Zhong $^{\mathrm{c}}$

a) Brunel Institute for Bioengineering, Brunel University, Uxbridge, Middlesex, UB8 3PH, UK

b) Wolfson Centre for Materials Processing, Brunel University, Uxbridge, Middlesex, UB8 3PH, UK

c) School of Materials Science and Engineering, Tianjin University, Weijin Road 92, Nankai District, Tianjin 300073, P.

R. China

* Corresponding author: Tel.:+44 1895 266123; Fax: +44 1895 269737; E-mail: wenhui.song@ @runel.ac.uk;

$†$ Present address: c) Electrical Engineering Division, Department of Engineering, University of Cambridge, CAPE Building, Cambridge, CB3 0FA, UK

\begin{abstract}
A novel brush-like electrode based on carbon nanotube (CNT) nano-yarn fiber has been designed for electrochemical biosensor applications and its efficacy as an enzymatic glucose biosensor demonstrated. The CNT nano-yarn fiber was spun directly from a chemical-vapor-deposition (CVD) gas flow reaction using a mixture of ethanol and acetone as the carbon source and an iron nano-catalyst. The fiber, $28 \mu \mathrm{m}$ in diameter, was made of bundles of double walled CNTs (DWNTs) concentrically compacted into multiple layers forming a nano-porous network structure. Cyclic voltammetry study revealed a superior electrocatalytic activity for CNT fiber compared to the traditional Pt-Ir coil electrode. The electrode end tip of the CNT fiber was freeze-fractured to obtain a unique brush-like nano-structure resembling a scale-down electrical 'flex', where glucose oxidase (GOx) enzyme was immobilized using glutaraldehyde crosslinking in the presence of bovine serum albumin (BSA). An outer epoxy-polyurethane (EPU) layer was used as semi-permeable membrane. The sensor function was tested against a standard reference electrode.
\end{abstract} The sensitivities, linear detection range and linearity for detecting glucose for the miniature CNT fiber electrode were better than that reported for a Pt-Ir coil electrode. Thermal annealing of the $\mathrm{CNT}$ fiber at $250{ }^{\circ} \mathrm{C}$ for 30 min prior to fabrication of the sensor resulted in a 7.5 fold increase in glucose sensitivity. The as-spun CNT fiber based glucose 
biosensor was shown to be stable for up to 70 days. In addition, gold coating of the electrode connecting end of the CNT fiber resulted in extending the glucose detection limit to $25 \mu \mathrm{M}$. To conclude, superior efficiency of CNT fiber for glucose biosensing was demonstrated compared to a traditional Pt-Ir sensor.

\section{Introduction}

In application as biosensors, CNTs demonstrate faster response time and higher sensitivity than traditional electrodes [1-3]. The better performance is attributed to their one-dimensional hollow tubular nano-chemistry that is responsible for the efficient capture and promotion of electron transfer reactions from analytes. The immense potential for CNT based materials for electrochemical sensing applications, especially clinical diagnostics and environmental monitoring, is widely recognized [1]. However, their integration into biosensing electrodes has been challenging and concerns are often raised regarding toxicity of the nano-sized CNTs leaching from an implanted biosensor [4-8].

Individual multiwall CNTs in a suspension have been proven to be cytotoxic [4]. However, several studies report that cytotoxicity is avoided by immobilizing CNTs on surfaces or within composites [5]. Hence, the development of CNTs in non-particulate forms, such as CNT paste, composite, films, thin coatings or continuous fibers, is considered a safe way to fabricate electrodes.

Early studies for integration of CNTs in electrodes concentrated on the use of CNTs as intermediates between electrodes and enzymes. Randomly dispersed CNTs were coated onto Pt, Au or glassy carbon electrodes using a binder to form an intermediate CNT paste [9]. Another approach involved making composites with polymers such as Teflon and polyvinyl acetate [10]. To gain better control over the distribution and robustness of the CNT coat on electrodes, chemical means, such as self-assembly on gold surfaces using chemical binding via $\mathrm{Au}-\mathrm{S}$ bonds [11] and immobilization of the enzyme on CNT ensembles through carbodiimide chemistry [12] were employed. Nevertheless, challenges remained in the development of robust, practical and stable sensors capable of maximum exploitation of the exceptional properties of individual CNTs. In addition, there is the potential risk of CNT leaching from the CNT ensembles when implanted in the body. 
The alignment of nanotubes in the fiber is an effective way to exploit the exceptional anisotropic properties of individual CNTs and transform them for micro/macroscale applications. Apart from good mechanical properties, the fiber inherits the advantages of the high surface area and good electrocatalytic properties of CNTs, while avoiding the potential toxicity caused by individual CNTs in the form of particles with a high aspect ratio during implantation. In recent years, several production routes were developed to fabricate continuous CNT micro-fibers, including wet spinning of CNTs from polymer dispersions or acid dispersions, dry spinning from aligned CNT matrices and direct spinning from CVD reactions [13-19].

Wang et al [20] first introduced wet-spun CNT fiber as a microelectrode for electrochemical sensors, and demonstrated the possibility of detection of NADH, hydrogen peroxide and dopamine in 2003. The CNT fiber was proven to have higher electrocatalytic activities when compared to a carbon fiber. The immobilization of mediators (2,4,7-trinitro-9-fluorenone) on the surface and the effect of pre-treatment of the same fiber on the performance of the biosensor were reported later [21, 22]. However, the wet-spun fibers from polymer solution are actually composite fibers consisting of non-conductive polymer as-spun, or carbon residue after thermal treatment, showing relatively low strength and low conductivity compared to pure CNT fibers.

Recently, a wide variety of continuous yarns of CNTs were fabricated by direct spinning of pure CNT fibers from an aerogel formed during a CVD process using ethanol and acetone as the carbon source [17-19]. The resulting CNT micro-fiber has the potential to address the electrode design and toxicity concerns of CNT based sensors for long-term implantable biosensor applications. During the CVD process, the CNTs form and self-assemble in the gas flow by van der Waals interactions at high temperature, and are then spun into nano-yarns along the fiber axis. Direct spinning of the pure CNT fiber by the CVD method helped to achieve, so far, the best properties in terms of strength, stiffness, toughness, as well as electrical and thermal conductivities in comparison with the CNT fibers spun from other methods [13-16]. The intention of our studies is to exploit the exceptional properties of a pure CNT fiber that is directly spun during CVD for biosensing. The specific fiber we are investigating is composed of double walled CNTs that are 
compacted into concentric layers of CNT bundles organized as nano-yarns [19]. The CNT fiber resembles an electric wire, except that it has a nanoscale surface topography and porosity, which we hypothesize, would facilitate molecular-scale interactions with agents like enzymes aiding in the efficient capture and promotion of electron transfer reactions. The objective for this study is to test this hypothesis by immobilizing glucose oxidase on CNT fiber and utilizing as an amperometric glucose biosensor. Amperometric glucose biosensing has been extensively studied and, hence, would serve as an excellent reference to establish the efficacy of CNT fiber in biosensing.

Here, we put forward a brush-like electrode of CNT nanoyarn fiber for enzymatic glucose biosensing. The CNT fiber was fabricated by a CVD process and its nano-topography demonstrated using scanning electron microscopy (SEM) and atomic force microscopy (AFM). Cyclic voltammetry measurements were used to compare the electrocatalytic activity of the CNT fiber with that of the traditional Pt electrode. The electrode end of the fiber was freeze-fractured to mechanically unwind the nano-yarn into a brush-like end. Glucose oxidase (GOx) was immobilized on the brush-like electrode end. The resulting CNT fiber based glucose biosensor was tested for glucose sensing functional efficacy in buffers. This study demonstrated the CNT fiber based sensor, having much smaller electrode size, performed better than a Pt-Ir coil based sensor. In addition, the improvement of surface conductivity of the CNT fiber, at its electrode connecting end, by gold coating revealed the fiber's wider range of analyte detection limits compared to noble metal electrodes.

\section{Experimental details}

\subsection{Materials}

Bovine serum albumin (BSA), glutaraldehyde (50\% v/v), polyurethane (PU), ATACS5104 epoxy adhesive, potassium ferricyanide, potassium chloride, glucose, dextrose, bovine adult serum, glucose oxidase (GOx) (EC1.1.3.4, Type X-S, Aspergillus niger, $157500 \mathrm{U} \mathrm{g}^{-1}$, Sigma), tetrahydrofuran (THF), polyethylene glycol dodecyl ether (Brij 30) were purchased from Sigma-Aldrich-Fluka. All glucose solutions were prepared from a phosphate buffer (PBS) with the 
ionic strength of $0.15 \mathrm{M}\left(0.025 \mathrm{M} \mathrm{Na}_{2} \mathrm{HPO}_{4}, 0.025 \mathrm{M} \mathrm{KH}_{2} \mathrm{PO}_{4}\right.$ and $\left.0.15 \mathrm{M} \mathrm{NaCl}\right)$. Teflon-covered platinum-iridium (9:1 in weight) wire (125 $\mu \mathrm{m}$ in diameter) was obtained from World Precision Instruments, Inc. (Sarasota, FL). ATACS 5104 is a fast cured two-component adhesive composed of modified mercaptan curing agent (part A) and epoxy resin blend (part B) including 50-75\% epoxy novolac resin, 1-5\% bisphenol-A epoxy resin and 20-50\% aliphatic triglycidyl ether.

\subsection{Synthesis of CNT nano-yarn fiber}

The continuous CNT nano-yarn fiber was spun directly from a chemical-vapor-deposition (CVD) gas flow reaction using a mixture of ethanol and acetone as the carbon source and an iron nano-catalyst according to the protocol reported earlier [19]. Briefly, the synthesis was conducted by the injection of the carbon source dispersed with ferrocene and thiophene into a heated gas flow reactor in flowing hydrogen. The CNTs were generated in the heated chamber and, with the assistance of gas flow, organized into continuous, concentric and discrete layers referred to as a multilayered CNT 'sock'. Initiated from the upper gas flow, the CNT sock traveled downstream into a water tank connected at the end of the CVD reactor. Upon arriving at the water surface, the CNT sock shrank into a fiber, which was collected around a rotator in water. Then the fiber was further washed and shrunk in acetone. Subsequently, the fiber was dried by an infrared heater at $\sim 100{ }^{\circ} \mathrm{C}$, wound onto a spool and stored at room temperature until further use.

\subsection{Fabrication and characterization of CNT fiber electrode}

About $70 \mathrm{~mm}$ long CNT fibers were used for the fabrication of electrodes. Some of the fibers were thermally annealed at $250^{\circ} \mathrm{C}$ for $30 \mathrm{~min}$ to improve their purity and electrochemical performance. Both thermally annealed and as-spun fibers were used to fabricate electrodes for CNT fiber based glucose biosensors, to test the effect of thermal treatment on the function of the biosensor. The sensing element end of the fiber was quenched in liquid nitrogen, fractured and nano-yarn manually unwound to obtain a brush-like end. The brush-like end was intended to increase the surface area for enzyme coating. 
A coil-type metal electrode, based on Pt-Ir, was prepared as reported earlier [23, 24] and used as the control to test the efficacy of CNT fiber as an electrode for electrochemical sensing. Briefly, a $10 \mathrm{~mm}$ long Teflon coating stripped end of $70 \mathrm{~mm}$ long, $125 \mu \mathrm{m}$ diameter Pt:Ir (9:1) wire was wound along a 30-gauge needle to form a cylindrical coil having an outer diameter of $0.55 \mathrm{~mm}$ and an inner diameter of $0.3 \mathrm{~mm}$.

\subsubsection{Morphology of the CNT fiber electrode.}

The morphology of the CNT fiber electrode was evaluated using scanning electron microscopy (SEM) and atomic force microscopy (AFM). A Zeiss Supra 35VP (Germany) field emission SEM (FESEM) in SE mode without any sample pre-treatment and a Digital Instruments Nanoscope III (USA) atomic force microscope (AFM) in tapping mode were used to record SEM photomicrographs and AFM topography images respectively.

\subsubsection{Cyclic voltammetry.}

Cyclic voltammetry experiments (CVs) were carried out with a computer-controlled electrochemical analyzer (Potentiostat 263A, Princeton Applied Research, US). The typical cyclic voltammograms from the asspun and annealed CNT fibers and coil-type Pt-Ir electrodes were obtained in $4 \mathrm{mM} \mathrm{K}_{3} \mathrm{Fe}(\mathrm{CN})_{6}$ in $0.1 \mathrm{mM} \mathrm{KCl}$ solution at a 50 $\mathrm{mV} \mathrm{s}^{-1}$ scanning rate.

\subsection{Fabrication of glucose sensor based on CNT fiber electrode}

The CNT fiber electrode was inserted into an insulating silastic tubing (Dow Corning, I.D. $1 \mathrm{~mm}$, length: $30 \mathrm{~mm}$ ), which also improved the handling of the microelectrode. The two ends of the tubing were sealed by a resin adhesive (R.S, UK) such that the two CNT fiber tips were left protruding by $1 \mathrm{~mm}$ (brushlike tip end) and 5 mm (electrode connecting end).

Glucose oxidase (GOx) was immobilized directly on the brush-like electrode end by solution casting. Fresh enzyme loading solution, containing $2.5 \mathrm{mg} \mathrm{GOx}, 12 \mathrm{mg} \mathrm{BSA}$ and $5 \mu \mathrm{l}$ of 50\% glutaraldehyde solution dissolved in $300 \mu \mathrm{l}$ of deionized water, was cast twice at the brush-like end of the CNT fibers (1.5-2.5 $\mu$ l each time). The enzyme loading 
solution was allowed to dry and crosslink at room temperature for about $60 \mathrm{~min}$ and overnight, respectively, after the first and second solution casts, respectively. The morphology of the enzyme loaded CNT fiber electrode end coated with GOx was examined using FESEM, as presented in section 2.3.1.

To extend the linear measuring range (covering 2-30 mM) of the glucose sensor, an EPU semi-permeable membrane is solvent cast on the miniature sensing element [23, 24]. A $5 \mu$ l aliquot of EPU coating solution containing $26.7 \mathrm{mg}$ of PU, $17.8 \mathrm{mg}$ of epoxy resins part A \& B each, $1 \mu \mathrm{l}$ of Brij 30 (surfactant) dissolved in $4 \mu \mathrm{l}$ of tetrahydrofuran (THF), was solution cast over the enzyme coating. After air-drying for $1 \mathrm{~h}$, EPU layer was cured at $80 \circ \mathrm{C}$ for $20 \mathrm{~min}$.

The lead end, used for connecting to the potentiostat, of some of the sensors was coated with a 30 nm layer of gold to test its enhancing effect on the electrochemical behavior of the CNT fiber based glucose sensors.

\subsection{Sensor function testing}

The function of the sensors was tested by amperometric measurements of glucose in buffers using potentiostats (Apollo 4000 Free Radical Analyzer, World Precision Instruments, US) at 0.7 V versus a saturated calomel electrode (SCE) as the reference electrode and a platinum mesh electrode as the counter-electrode. All potentials were measured and reported versus the SCE. The response time, sensitivity and linear range of the sensors, as a function of time, was determined. The response time represents the time required to reach $90 \%$ of the maximum response when the glucose concentration increases from 5 to $15 \mathrm{mM}$, while sensitivity was determined by a two-point method (between 5 and 15 $\mathrm{mM}$ glucose solutions) by calculating the sensor's response current (nA) per mM glucose concentration. Calibration plots for each sensor were obtained by stepwise addition of $100 \mathrm{mM}$ glucose solution to $8 \mathrm{ml}$ phosphate buffer saline (PBS, pH 7.4) covering 2-30 mM. All experiments were carried out at room temperature $\left(24 \pm 1{ }^{\circ} \mathrm{C}\right)$. The long-term stability of the CNT fiber based glucose sensor was investigated by storing the sensors in $5 \mathrm{mM}$ glucose solution in PBS ( $\mathrm{pH} 7.4)$ at $24 \pm 1 \circ \mathrm{C}$. The glucose solution was changed every 2 days, and the sensors tested for sensitivity every 2-5 days over a period of 90 days, to record the changes in sensitivity as a function of time. The stability of CNT fiber 
biosensors was also tested after storage in PBS solution at $4{ }^{\circ} \mathrm{C}$ for 3 months. (The storage solution was replaced with fresh PBS every 2-5 days.)

\section{Results and discussion}

High purity (as high as 96.6\%) CNT micro-fibers have recently been developed in the form of continuous nano-yarns [19]. The fiber consists of multiple mono-layers of a CNT bundles (i.e. nano-yarns) network that are concentrically assembled in seamless tubules along the yarn axis. In the current study, we utilized one such CNT fiber as an electrode for glucose biosensing.

\subsection{The CNT fiber}

The SEM images illustrated in figure 1 show the morphology of the CNT fiber. The fiber has an average diameter of $28 \mu \mathrm{m}$ and is slightly twisted along the fiber axis (figure 1(a)). The slight twisting could be due to the spinning process. At a higher magnification (figure 1(b)), the fiber's surface nano-topography is evident. CNT bundles of about $50 \mathrm{~nm}$ in diameter are entangled to form a nano-porous mesh-like surface topography. The AFM image in figure 2 further confirms the surface nano-topography consistent with the SEM image in figure 1(b). The fiber has a nano-structural hierarchy, which, along with the CNT fiber's physical properties, has recently been reported by Zhong et al [19].

Briefly, the basic structural unit, CNT, is organized into DWNTs having a diameter of 8-10 nm. The DWNTs further assemble into $\sim 50 \mathrm{~nm}$ bundles that correspond to the nanoyarns observed in the SEM and AFM images (figures 1(b) and 2). Zhong et al [19] demonstrated that the nano-yarns are organized into discrete and concentric mono-layers of $\sim 50 \mathrm{~nm}$ CNT bundles held together by molecular forces which include van der Waal interactions. The schematic organization of the mono-layers of CNT bundles into the CNT micro-fiber is illustrated in figure 3. The degree of alignment of the CNT bundles along the acetone densified yarn axis is reported to be 0.86 (as determined by wide angle X-ray diffraction). Brunauer-Emmett-Teller (BET) and Barrett-Joyener-Halenda (BJH) measurements respectively demonstrated that the surface of the CNT yarn has a high specific surface area of $194 \mathrm{~m}^{2} \mathrm{~g}^{-1}$ and an average pore size around $2.4 \mathrm{~nm}$. The multilayered yarn has a tensile strength in the range of $0.4-1.25 \mathrm{GPa}$ and exhibits good elastic behavior and resistance to cyclic stretching. In addition to superior mechanical properties, the CNT fiber has an electrical conductivity, $5.0 \times 10^{5} \mathrm{~S} \mathrm{~m}^{-1}$, two orders of magnitude higher than that of wet-spun CNT fibers $\left((1-2) \times 10^{3} \mathrm{~S}\right.$ $\left.\mathrm{m}^{-1}\right)[13,20]$.

The multiple coaxial bundles of CNTs in compacted seamless tubules spanning the length of the fiber render a polymeric macromolecular structure and effectively prevent CNT cytotoxicity. The macro-length structure, combined with high elasticity, toughness and light weight, potentially makes the CNT fiber an inert biocompatible material when implanted in the body. Furthermore, the exceptionally high electrical conductivity $\left(5.0 \times 10^{5} \mathrm{~S} \mathrm{~m}^{-1}\right)$, surface area (>194 
$\mathrm{m}^{2} \mathrm{~g}^{-1}$ ) and nano-topography aspects are anticipated to make the CNT fibers suitablematerials for implantable biosensor applications.

\subsection{The CNT fiber electrode}

Due to the 1D electronic structure of CNTs, electronic transport is reported to occur ballistically (without scattering) over long nanotube lengths, enabling CNTs to carry high currents with essentially no heating [25]. Hence, to realize the full potential of the CNT fiber as a biosensor, it was essential to unwind the CNT bundles (nano-yarns) at the ends of the fibers. The separated nano-yarns at the end tip of the CNT fiber, shown in figure 4, are prepared by manual tweezing of freeze-fractured ends. The resulting brush-like nano-structure resembles a scaled-down electrical 'flex' and the individual nano-yarns within the brush-like end would act as multi-nano-electrodes, reminiscent of a dendrite-type nerve cell, which provide many nano-channels to promote and speed up electron transfer as well as increase the surface area for enzyme immobilization.

Regardless of its high CNT content, CNT fiber spun directly from the CVD gas flow reaction inherits impurities such as metal (iron) nano-catalyst, amorphous carbon or graphite. The presence of such impurities in the CNT fiber is evident from the thermo-gravimetric analysis result reported by Zhong et al [19], and it is known that impurities can significantly affect the electronic properties of the CNT based materials [25, 26]. Nevertheless, thermal treatment of the CNT materials is reported to remove the majority of amorphous and crystalline carbon as well as the surface-adsorbed impurities, effectively enhancing both the electrochemical activity and the purity of a CNT based material $[26,27]$. To further study the effects of thermal treatment on CNT fiber as a glucose biosensor, we annealed some of the fibers in air at $250{ }^{\circ} \mathrm{C}$ just prior to the fabrication of the electrode. Surface characterization of our CNT fibers before and after the thermal treatment using SEM, in fact, reveals a smoother CNT surface with little debris (data not shown). In addition, the increase in electrical conductivity is demonstrated by 7.5 fold increase in sensitivity of the glucose sensors prepared using heat annealed CNT fibers (details in section 3.4). Thus our studies further confirm the effect of thermal treatment on enhancement of the electrical conductivity of CNT based materials.

For fabrication of the glucose biosensor, first, the length of the CNT fiber, except $1 \mathrm{~mm}$ at the sensing end and $5 \mathrm{~mm}$ at the electrode connecting end, was insulated using silicon tubing, with the tubing ends sealed by epoxy resin. In addition to insulation, the silicon tubing also enhanced the physical handling of the lightweight CNT fiber. Thereafter, at the brush-like sensing end of the electrode, GOx enzyme was immobilized by glutaraldehyde crosslinking of GOx on a BSA matrix. The morphology of GOx immobilized on the circumferential surface of the CNT fiber is shown in figure 5. The immobilized GOx-BSA crosslinked network layer appears to fit snugly to the contours of the nano-porous surface topography of the CNT fiber surface. Because of the $\mathrm{sp}^{2}$ carbon atoms in the polycyclic aromatic hydrocarbon 
chemistry of the graphene tubes in CNTs and the exclusive Schiff's base glutaraldehyde crosslinking reaction of GOx-BSA proteins, covalent interactions between CNTs and GOx-BSA layers are not feasible. Hence, the GOx layer is expected to interact with CNT fiber non-covalently, which is consistent with reports that different enzymes, polypeptides and DNA can interact non-covalently with CNT [28-35]. The interactions between proteins and CNT are reported to involve many types of non-covalent forces, including electrostatic, hydrogen bonding, hydrophobic and $\pi-\pi$ interactions; and strong non-covalent interactions were also demonstrated between GOx and DWNTs [34]. Hence, strong molecular level non-covalent interactions between GOx and CNTs bundles, both at the fiber's porous surface and the separated nano-yarns of our fiber's brush-like end tip, are expected to provide active catalytic coupling of the enzyme redox reaction directly with the CNT surface (figure 3).

\subsection{Electrochemical characterization}

The electrochemical signal transduction ability of the CNT fiber as microelectrodes is characterized using traditional cyclic voltammetry, and compared with a Pt-Ir coil electrode. Figure 6 illustrates the typical cyclic voltammograms from Pt-Ir coil, as-spun and annealed CNT fiber electrodes in $4 \mathrm{mM} \mathrm{K}_{3} \mathrm{Fe}(\mathrm{CN})_{6}$ in $0.1 \mathrm{mM} \mathrm{KCl}$ solution at a $50 \mathrm{mV} \mathrm{s}^{-1}$ scanning rate. The well-defined oxidation and reduction peaks due to the $\mathrm{Fe}^{3+} / \mathrm{Fe}^{2+}$ redox couple for the annealed CNT fiber electrode are significantly higher compared to the Pt-Ir coil and as-spun CNT fiber electrode. The corresponding results presented in table 1 show that the anodic peak current $\left(I_{p}\right)$ of the annealed CNT fiber $\left(23.25 \mu \mathrm{A} \mathrm{mm}{ }^{-2}\right)$ is about double that of the Pt-Ir coil electrode $\left(11.3 \mu \mathrm{A} \mathrm{mm}^{-2}\right)$ and as-spun CNT fiber $\left(9.8 \mu \mathrm{Amm}^{-2}\right)$.

The electro-active surface area of the electrode, A, can be calculated by fitting the above cyclic voltammetry results in the modified Randles-Sevcik equation [36, 37]:

$$
I_{p}=2.69 \times 10^{5} A D^{1 / 2} N^{3 / 2} \gamma^{1 / 2} C
$$

where $A$ is the electro-active area of the electrode $\left(\mathrm{cm}^{2}\right), D$ is the diffusion coefficient of the molecule in solution $\left(\mathrm{cm}^{2}\right.$ $\left.\mathrm{s}^{-1}\right), N$ is the number of electrons participating in the redox reaction, $\gamma$ is the scan rate of the potential $\left(\mathrm{V} \mathrm{s}^{-1}\right)$, and $C$ is the concentration of the probe molecule in the bulk solution $\left(\mathrm{mol} \mathrm{cm}{ }^{-3}\right)$. The $\mathrm{Fe}(\mathrm{CN})_{6}^{4-} /^{3-}$ redox system used in this study is one of the most extensively studied redox couples in electrochemistry and exhibits a heterogeneous one-electron transfer $(\mathrm{N}=1) . C$ is equal to $4 \mathrm{mM}$, and the diffusion coefficient $(D)$ is $6.7 \times 10^{-6} \mathrm{~cm}^{2} \mathrm{~s}^{-1}$, while the scan rate $(\gamma)$ is $50 \mathrm{mVs}^{-1}$. According to equation (1), the electroactive surface area for as-spun CNT fiber is $0.002 \pm 0.001 \mathrm{~cm}^{2}$ $( \pm \mathrm{SD}, \mathrm{n}=3)$. Nevertheless, the annealed CNT fiber is $0.006 \pm 0.001 \mathrm{~cm}^{2}( \pm \mathrm{SD}, \mathrm{n}=3)$, which is about six times larger than its apparent geometric area $0.0009 \mathrm{~cm}^{2},(28 \mu \mathrm{m}$ in diameter, $1 \mathrm{~mm}$ in length). In contrast, the electro-active surface area for the platinum coil $\left(125 \mu \mathrm{m}\right.$ ca. Pt-Ir wire) appears much larger, about $0.031 \pm 0.002 \mathrm{~cm}^{2}( \pm \mathrm{SD}, \mathrm{n}=3)$. However, it is only ca. $63 \%$ of its apparent geometric area $0.0491 \mathrm{~cm}^{2}(125 \mu \mathrm{m}$ in diameter, $1.25 \mathrm{~cm}$ in length). The substantially 
high effective electro-active surface area of CNT fiber with respect to its apparent geometric area for CNT fiber is attributed to the nano-porous surface topography of CNT nano-yarns, as shown in figures 1(b) and 2. Furthermore, the potential difference $\Delta E_{p}$ between the anodic and cathodic peak potentials is 90 and $93 \mathrm{eV}$ respectively for the as-spun and annealed CNT fibers, which is nearly half that for the Pt coil, $177 \mathrm{eV} . \Delta E_{p}$ is reported to be directly related to the electron transfer rate [28]. The lower $\Delta E_{p}$ of the CNT fiber signifies that the electron transfer rate for the CNT fiber is faster than that for the Pt coil electrode. These results indicate that the CNT fiber electrode has superior electrocatalytic activities compared with the Pt coil electrode. Thus, in combination with its excellent affinity with enzymes and other biomolecules, CNT fiber is an attractive material to replace traditional noble metal electrodes $(\mathrm{Pt}$ and $\mathrm{Au})$ for electrochemical biosensor applications.

\subsection{Amperometric response of the biosensor}

Figure 7 shows the typical amperometric response of the CNT fiber based glucose biosensor. The fast response of the biosensor toward glucose can be seen, in that the sensor response current reaches a dynamic equilibrium within tens of seconds (response time) of each addition of glucose, generating a near steady-state current signal. This indicates a fast electron transfer between the redox center of the enzyme and the CNT fiber, as described by Cai et al [3].

The linear calibration curves for the annealed and asspun CNT fiber glucose biosensors are presented in figure 8. Both the sensors showed a linear increase $\left(\mathrm{R}^{2}\right.$ of 0.991 and 0.997 respectively for annealed and as-spun CNT fibers) in sensor response current covering the $2-30 \mathrm{mM}$ of glucose concentration range desired for sensing covering the whole range of physiological blood glucose levels.

The sensitivity, S, of the sensors was determined by using a two-point method described in equation (2) as follows [23, 24],

$$
S=\left(I_{2}-I_{1}\right) / 10
$$

where I1 and I2 represent the sensor response currents obtained in $5 \mathrm{mM}$ and $15 \mathrm{mM}$ glucose solutions respectively. The sensitivity for the annealed CNT fiber sensor $\left(7.2 \mathrm{nM} \mathrm{mM}^{-1}\right)$ was significantly higher than that for the unannealed CNT fiber sensor $\left(0.96 \mathrm{nA} \mathrm{mM}^{-1}\right)$ (figure 8), indicating that the conductivity of the CNT fiber increased significantly following thermal treatment (annealing). Considering that the long-term storage of CNT based materials leads to a proportional decrease in conductivity, due to inherent impurities within the as-spun fibers as well as spontaneous coating of the CNT surface with impurities $[25,26]$, thermal annealing of the CNT fiber just prior to biosensor fabrication is a key aspect for fabrication of a CNT fiber based biosensor.

The CNT fiber based glucose biosensor was coated with a similar volume and composition of GOx loading solution to the Pt coil sensor, as reported by Yu et al [24], except that the sizes of the sensing element differed. While the Pt coil 
used by Yu et al [24] was $\sim 10 \mathrm{~mm}$, our CNT fiber's sensing element was kept to $\sim 1 \mathrm{~mm}$ in length, including the brush-like end shown in figure 4. Effectively, a similar amount of GOx loading solution was applied to effective electro-active surface areas of $0.031 \pm 0.002 \mathrm{~cm}^{2}$ for a $\sim 10 \mathrm{~mm}$ long Pt coil compared to $0.002 \pm 0.001$ and $0.006 \pm$ $0.001 \mathrm{~cm}^{2}$ for $\sim 1 \mathrm{~mm}$ long as-spun and annealed CNT fiber based sensors respectively. Yu et al reported that the Pt coil based glucose biosensors had a sensitivity ranging between 1 and $6 \mathrm{nA} \mathrm{mM}{ }^{-1}$, linearity in the range of $1-25 \mathrm{mM}$, and $\mathrm{R}^{2}>0.998$ [24]. The unannealed and annealed CNT fiber based biosensors had sensitivities of 0.96 and $7.2 \mathrm{nA} \mathrm{mM}^{-1}$ respectively. The linear detection range and $\mathrm{R}^{2}$ are also comparable, although the sensor response time (90\%) for CNT based sensors $(\sim 10 \mathrm{~s})$ is much faster than that reported for Pt-Ir coil sensors $(20-200 \mathrm{~s})$. These results suggest that the miniature CNT electrode $(\sim 1 \mathrm{~mm})$ performs better than the reported $\sim 10 \mathrm{~mm}$ long Pt coil electrode when tested as a glucose biosensor.

The long-term stability of the unannealed CNT fiber based glucose sensors was tested over a period of 90 days by two methods. In the first method, the sensors were stored in $5 \mathrm{mM}$ glucose solution in PBS at room temperature $\left(24 \pm 1{ }^{\circ} \mathrm{C}\right)$, and the sensitivity of the sensors tested every 2-5 days. The change in sensitivity as a function of time is illustrated in figure 9. The second method involved the storage of the sensor at $4{ }^{\circ} \mathrm{C}$ in $\mathrm{PBS} \mathrm{pH} 7.4$ and the sensor's sensitivity tested after 90 days. The initial increase in sensitivity up to about 10 days could be due to the swelling of the EPU and GOx-BSA layers (figure 9). The higher the swelling, the higher the water content in the respective layers, which in turn increases the amount of glucose diffusing to the enzyme layer, resulting in an increase in sensitivity. The sensitivity remained nearly constant from 10 to 70 days and, thereafter, a gradual reduction in sensitivity was observed until the end of the study period of 90 days (figure 9). Build-up of $\mathrm{H}_{2} \mathrm{O}_{2}$ within the enzyme layer during storage in $5 \mathrm{mM}$ glucose solution in PBS could be a reason for the decrease in sensitivity, because $\mathrm{H}_{2} \mathrm{O}_{2}$ was earlier demonstrated to deleteriously affect the function of GOx enzyme [38]. To circumvent this problem, excess loading of GOx was suggested [23], and our CNT fibers were loaded with excess enzyme per unit electro-active surface area. Furthermore, a similar extent of decrease in sensitivity was also observed for the batch of sensors that were stored in PBS (without glucose) at 90 days of storage. This suggests that even without significant accumulation of $\mathrm{H}_{2} \mathrm{O}_{2}$ within the enzyme layer $\left(\mathrm{H}_{2} \mathrm{O}_{2}\right.$ accumulation is expected to be maximal in the presence of glucose) there is a decrease in sensitivity. Hence, there are other significant factors that affect the function of the CNT fiber based glucose biosensor and require further investigation. One factor could be the accumulation of impurities on the CNT fiber connection end over the period of storage, due to the high affinity of the CNT fiber surface to a wide variety of molecules, including oxygen in air, resulting in a reduction in their surface conductivity [26].

The conductivity and nature of electron transfer in CNT fiber is significantly different from the metal connecting lead 
of the potentiostat, which could affect the function of the CNT fiber based glucose biosensor. During our experiments, we noticed that the biosensor performance was very sensitive to the electrode connection with the testing lead. The large contact resistance has been an issue for carbon nanotube sensors, electronics and devices [25]. To improve the contact between the CNT fiber and the connecting lead, we coated the CNT fiber's connecting end with a thin layer of gold ( $30 \mathrm{~nm}$ in thickness). The annealed CNT fiber sensors with gold coated connecting end were tested for sensitivity and were observed to be similar $\left(\sim 6.8 \mathrm{nAmM}^{-1}\right)$ to that of non-gold coated annealed CNT fiber sensors $\left(\sim 7.2 \mathrm{nAmM}^{-1}\right)$. In spite of finding no statistically significant difference between gold coated and non-gold coated connecting leads, we believe that the full potential of the CNT fiber's excellent conductivity can be realized with other appropriate lead connection intermediates and needs further investigation. However, an interesting phenomenon was observed when the CNT fiber's connecting end was coated with gold. It had the ability to detect glucose levels as low as $25 \mu \mathrm{M}$, which was not possible with the non-gold coated CNT fiber or the Pt coil based glucose biosensors. Thus, the range of the steady-state current in response to the glucose concentration extends to a range of $25 \mu \mathrm{M}$ to $30 \mathrm{mM}$, which is much wider than that of the carbon nano-fiber biosensor reported by Vamvakaki et al [39]. Such wider ranges of analyte detection limits, compared to conventional metal electrodes, could be of great use in applications such as environmental monitoring and drug testing for athletes and vehicle drivers, and further investigations are required to maximize the performance of the CNT fiber sensors through improvement of the electrode connection in reducing the surface resistance between the CNT fiber and the connecting leads.

\section{Summary}

In this study, the efficacy of the CNT fiber as an electrode and glucose biosensor is compared with that of a Pt-Ir coil-type electrode, where the Pt-Ir wire is about 4.5 times larger in diameter than the CNT fiber. The CNT fiber has a nano-scale topography having nano-fibers (bundles) forming a nano-yarn with nano-porosity. The anodic peak current for the miniature annealed CNT fiber was double that measured for a ten times larger (geometric size) Pt-Ir coil. Similarly, the sensitivities, linear detection range and linearity for detecting glucose for the miniature CNT fiber electrodes were better than that reported for the Pt-Ir coil electrode. Furthermore, thermal annealing of the as-spun CNT fiber was shown to increase the sensitivity of the fiber based glucose biosensor by 7.5 times; while the sensitivity of the sensors was demonstrated to be stable up to 70 days of storage. Although the coating of the CNT fiber's lead end with gold did not significantly affect the sensor's sensitivity, it resulted in the extension of the lower glucose detection limit from $2 \mathrm{mM}$ to as low as $25 \mu \mathrm{M}$. The widening of the analyte detection limits can have implications for better sensors for environmental monitoring and low concentration drug testing. To conclude, this study has proved the better 
efficiency of CNT fiber as a glucose biosensor compared to a traditional metal electrode. Further studies would involve addressing the long-term stability of the CNT fiber based glucose biosensor and identifying the right intermediates for connecting the CNT fiber surface to the potentiostat's connecting lead in order to realize the full potential of the exceptional conductivity of CNT fiber for biosensing.

\section{Acknowledgments}

This research was supported by the Brunel Institute of Bioengineering and the Wolfson Centre for Materials Processing, Brunel University, grant (RG 2009/R1) from the Royal Society and grant (R01EB001640) from the National Institute of Health (NIH/NIBIB). We would like to thank Professor George Chen, the University of Nottingham, for enlightening discussion, Drs Leonid Paramonov and Dung Ly, Brunel University, for their assistance in drawing a double walled CNT and the referees' constructive comments.

\section{References}

[1] Gooding J J 2005 Nanostructuring electrodes with carbon nanotubes: a review on electrochemistry and applications for sensing Electrochim. Acta $\mathbf{5 0} 3049-60$

[2] Yun Y H et al 2008 Fabrication and characterization of carbon nanotube array electrodes with gold nanoparticle tips Sensors Actuators B 133 208-12

[3] Cai C X and Chen J 2004 Direct electron transfer of glucose oxidase promoted by carbon nanotubes Anal. Biochem. 332 75-83

[4] Poland C A et al 2008 Carbon nanotubes introduced into the abdominal cavity of mice show asbestos-like pathogenicity in a pilot study Nat. Nanotechnol. 3 423-8

[5] Hussain M A, Kabir M A and Sood A K 2009 On the cytotoxicity of carbon nanotubes Curr. Sci. 96 664-73

[6] Monteiro-Riviere N A, Nemanich R J, Inman A O, Wang Y Y Y and Riviere J E 2005 Multi-walled carbon nanotube interactions with human epidermal keratinocytes Toxicol. Lett. $155377-84$

[7] Pantarotto D, Briand J P, Prato M and Bianco A 2004 Translocation of bioactive peptides across cell membranes by carbon nanotubes Chem. Commun. 16-7 
[8] Schipper M L et al 2008 A pilot toxicology study of single-walled carbon nanotubes in a small sample of mice Nat. Nanotechnol. 3 216-21

[9] Valentini F, Amine A, Orlanducci S, TerranovaM L and Palleschi G 2003 Carbon nanotube purification: preparation and characterization of carbon nanotube paste electrodes Anal. Chem. 75 5413-21

[10] Wohlstadter J N et al 2003 Carbon nanotube-based biosensor Adv. Mater. 15 1184-7

[11] Liu Z F et al 2000 Organizing single-walled carbon nanotubes on gold using a wet chemical self-assembling technique Langmuir 16 3569-73

[12] Lin Y H, Lu F, Tu Y and Ren Z F 2004 Glucose biosensors based on carbon nanotube nanoelectrode ensembles Nano Lett. 4 191-5

[13] Vigolo B et al 2000 Macroscopic fibers and ribbons of oriented carbon nanotubes Science 290 1331-4 [14] Jiang K L, Li Q Q and Fan S S 2002 Nanotechnology: spinning continuous carbon nanotube yarns-carbon nanotubes weave their way into a range of imaginative macroscopic applications Nature 419801

[15] Ericson L M et al 2004 Macroscopic, neat, single-walled carbon nanotube fibers Science 305 1447-50

[16] Zhang M, Atkinson K R and Baughman R H 2004 Multifunctional carbon nanotube yarns by downsizing an ancient technology Science 306 1358-61

[17] Li Y L, Kinloch I A and Windle A H 2004 Direct spinning of carbon nanotube fibers from chemical vapor deposition synthesis Science 304 276-8

[18] Koziol K et al 2007 High-performance carbon nanotube fiber Science 318 1892-5

[19] Zhong X-H et al 2010 Continuous multilayered carbon nanotube yarns Adv. Mater. 22 692-6

[20] Wang J, Deo R P, Poulin P and Mangey M 2003 Carbon nanotube fiber microelectrodes J. Am. Chem. Soc.

$12514706-7$

[21] Viry L, Derre A, Garrigue P, Sojic N, Poulin P and Kuhn A 2007 Carbon nanotube fiber microelectrodes: design, characterization, and optimization J. Nanosci. Nanotechnol. 7 3373-7 
[22] Viry L, Derre A, Garrigue P, Sojic N, Poulin P and Kuhn A 2007 Optimized carbon nanotube fiber microelectrodes as potential analytical tools Anal. Bioanal. Chem. 389 499-505

[23] Yu B Z, Moussy Y and Moussy F 2005 Coil-type implantable glucose biosensor with excess enzyme loading Front. Biosci. 10 512-20

[24] Yu B Z, Long N, Moussy Y and Moussy F 2006 A long-term flexible minimally-invasive implantable glucose biosensor based on an epoxy-enhanced polyurethane membrane Biosens. Bioelectron. 21 2275-82

[25] Baughman R H, Zakhidov A A and de Heer W A 2002 Carbon nanotubes - the route toward applications Science $297787-92$

[26] Collins P G, Bradley K, Ishigami M and Zettl A 2000 Extreme oxygen sensitivity of electronic properties of carbon nanotubes Science 287 1801-4

[27] Rinzler A G et al 1998 Large-scale purification of single-wall carbon nanotubes: process, product, and characterization Appl. Phys. A 67 29-37

[28] Lu X B, Zhou J H, Lu W, Liu Q and Li J H 2008 Carbon nanofiber-based composites for the construction of mediator-free biosensors Biosens. Bioelectron. 23 1236-43

[29] Shin S R et al 2008 DNA-wrapped single-walled carbon nanotube hybrid fibers for supercapacitors and artificial muscles Adv. Mater. 20 466-70

[30] Badaire S et al 2005 Liquid crystals of DNA-stabilized carbon nanotubes Adv. Mater. $171673-6$

[31] Jeynes J C G, Mendoza E, Chow D C S, Watts P C R, McFadden J and Silva S R P 2006 Generation of chemically unmodified pure single-walled carbon nanotubes by solubilizing with RNA and treatment with ribonuclease A $A d v$. Mater. 18 1598-602

[32] Patolsky F, Weizmann Y and Willner I 2004 Long-range electrical contacting of redox enzymes by SWCNT connectors Angew. Chem. Int. Edn 43 2113-7

[33] Yan X B, Chen X J, Tay B K and Khor K A 2007 Transparent and flexible glucose biosensor via layer-by-layer 
assembly of multi-wall carbon nanotubes and glucose oxidase Electrochem. Commun. 9 1269-75

[34] Pumera M and Smid B 2007 Redox protein noncovalent functionalization of double-wall carbon nanotubes:

electrochemical binder-less glucose biosensor J. Nanosci. Nanotechnol. 7 3590-5

[35] Wang J et al 2007 Electrocatalytic detection of insulin at RuOx/carbon nanotube-modified carbon electrodes Anal.

Chim. Acta 581 1-6

[36] Bard A J and Faulkner L R 2000 Electrochemical Methods: Fundamentals and Applications (New York: Wiley)

[37] Hrapovic S, Liu Y L, Male K B and Luong J H T 2004 Electrochemical biosensing platforms using platinum nanoparticles and carbon nanotubes Anal. Chem. 76 1083-8

[38] Valdes T I and Moussy F 2000 In vitro and in vivo degradation of glucose oxidase enzyme used for an implantable glucose biosensor Diabet. Technol. Ther. 2 367-76

[39] Vamvakaki V, Tsagaraki K and Chaniotakis N 2006 Carbon nanofiber-based glucose biosensor Anal. Chem. 78 5538-42 


\section{Figure Captions}

Figure 1. SEM photomicrograph showing the morphology of CNT fiber. (a) Microstructure of the CNT fiber showing the CNT bundles twisted along the longitudinal axis of the fiber. The fiber has an average diameter of $\sim 28 \mu \mathrm{m}$. (b) Surface nano-topography of the CNT fiber showing the individual CNT bundles (nano-fibers) entangled into a nano-yarn with a nano-porous network structure.

Figure 2. AFM photomicrograph showing the surface nano-topography of the CNT fiber. The nano-structure is consistent with the SEM photomicrograph, figure 1(b), showing the individual CNT bundles entangled into a nano-yarn with nano-porous network structure.

Figure 3. Schematic diagram showing (a) CNT fiber based glucose biosensor, (b) CNT bundles, (c) DWNT and (d) working principle of biosensor. The CNT fiber (ca. $28 \mu \mathrm{m})$ is made of bundles (ca. $~ 50 \mathrm{~nm}$ ) of DWNTs (ca. $8-10 \mathrm{~nm}$ ) entangled to form concentrically compacted multiple layers of nano-yarns along the CNT fiber axis, as illustrated in (a) and in [19]. GOx enzyme is immobilized at the brush-like end of the CNT fiber and the enzyme layer is encapsulated by the epoxy-polyurethane (EPU) semi-permeable membrane. D-glucose and oxygen pass through the EPU membrane, GOx converts them into gluconic acid and hydro peroxide. The peroxide is converted to electrons, hydrogen and oxygen at a potential of $700 \mathrm{mV}$. The electrons are captured by the fiber, the signal from which is used to measure glucose concentrations.

Figure 4. SEM photomicrograph showing the brush-like end of the CNT fiber prepared by snap freezing and twisting the end to unwind the nano-yarn. The separated individual CNT bundles are visible in the low (a) and high (b) magnification images.

Figure 5. SEM photomicrograph showing the morphology of glucose oxidase enzyme immobilized on the CNT fiber. The enzyme layer appears to be uniform and snugly immobilized on the surface, evident from the contours showing the ridges and grooves corresponding to the individual CNT bundles and the nano-pores between them.

Figure 6. Cyclic voltammograms of different electrodes: (1) Pt-Ir coil, (2) as-spun CNT fiber, (3) annealed CNT fiber. The curves were obtained in a solution of $4 \mathrm{mM} \mathrm{K}_{3} \mathrm{Fe}(\mathrm{CN})_{6}$ in $0.1 \mathrm{mM} \mathrm{KCl}$ at a $50 \mathrm{mV} \mathrm{s}^{-1}$ scan rate. The anodic 
peak currents for Pt-Ir coil, as-spun and annealed CNT fiber electrodes are 11.3, 9.8 and $23.3 \mu \mathrm{A} \mathrm{mm}^{-2}$ respectively; the cathodic peak currents are 15.2, 13.7 and $23.1 \mu \mathrm{Amm}^{-2}$ and potential differences between anodic and cathodic peaks $\left(\Delta E_{p}\right)$ are 177,90 and $93 \mathrm{eV}$, respectively.

Figure 7. A typical amperometric response curve for a CNT fiber based glucose biosensor. Numbers in the chart represent the corresponding glucose concentration of the solution. The near instantaneous rise in the sensor response current following the addition of aliquots of $100 \mathrm{mM}$ glucose stock solution corresponding to 2, 5, 10, 15, 20, 25 and 30 $\mathrm{mM}$ final test concentrations are evident from the stepwise increase in the sensor response curve.

Figure 8. Calibration curves of the annealed and as-spun CNT fiber based glucose biosensors $(n=3)$ showing linearity for the $2-30 \mathrm{mM}$ glucose solution concentration range.

Figure 9. Long-term sensitivity variation of as-spun CNT fiber based glucose biosensors. Between the sensitivity testing, the sensors were stored in a $5 \mathrm{mM}$ glucose PBS solution at $24 \pm 1{ }^{\circ} \mathrm{C}$. 
Table 1. Electrochemical properties of the Pt-Ir coil and CNT fiber based electrode based on the cyclic voltammetry results

\begin{tabular}{|l|c|c|c|c|c|}
\hline Electrode & $\begin{array}{l}\text { Anodic Peak } \\
\text { current }\left(I_{p}\right) \\
\left(\mu \mathrm{Am}^{-2}\right)\end{array}$ & $\begin{array}{l}\text { Cathodic } \\
\text { Peak current } \\
\left(\mu \mathrm{A} \mathrm{mm}^{-2}\right)\end{array}$ & $\begin{array}{l}\text { Potential difference } \\
\text { between anodic and } \\
\text { cathodic peak currents } \\
\left(\Delta E_{p}\right)(\mathrm{eV})\end{array}$ & $\begin{array}{l}\text { Electro-active } \\
\text { surface area } \\
\left(\mathrm{cm}^{2}\right)\end{array}$ & $\begin{array}{l}\text { Apparent } \\
\text { geometric } \\
\text { area }\left(\mathrm{cm}^{2}\right)\end{array}$ \\
\hline $\begin{array}{l}\text { Pt coil }(12.5 \mathrm{~mm} \text { long, } 125 \\
\mu \mathrm{m} \text { diameter })\end{array}$ & 11.3 & 15.2 & 177 & $0.031 \pm 0.002$ & 0.0491 \\
\hline $\begin{array}{l}\text { As-spun CNT fiber }(1 \mathrm{~mm} \\
\text { long, } 28 \mu \mathrm{m} \text { diameter })\end{array}$ & 9.8 & 13.7 & 90 & $0.002 \pm 0.001$ & 0.0009 \\
\hline $\begin{array}{l}\text { Annealed CNT fiber }(1 \mathrm{~mm} \\
\text { long, } 28 \mu \mathrm{m} \text { diameter) }\end{array}$ & 23.3 & 23.1 & 93 & $0.006 \pm 0.001$ & 0.0009 \\
\hline
\end{tabular}



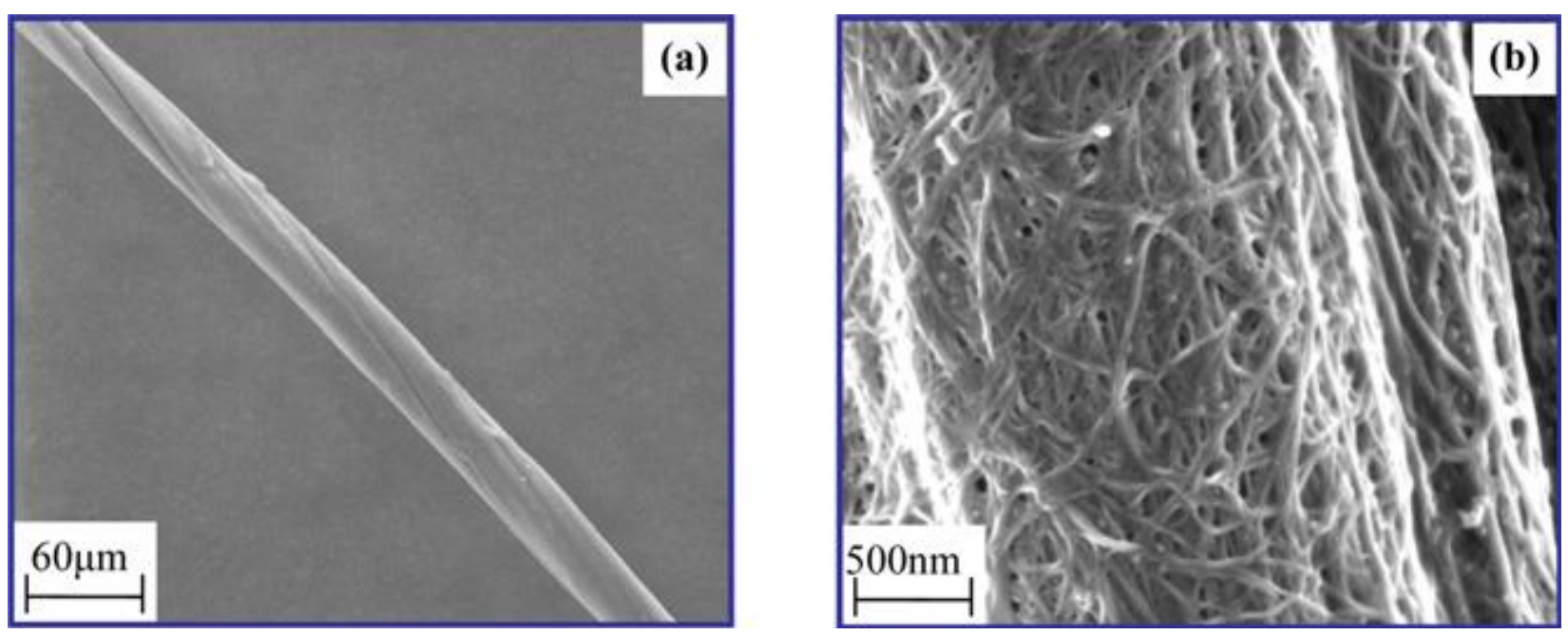

Figure 1. SEM photomicrograph showing the morphology of CNT fiber. (a) Microstructure of the CNT fiber showing the CNT bundles twisted along the longitudinal axis of the fiber. The fiber has an average diameter of $\sim 28 \mu \mathrm{m}$. (b) Surface nano-topography of the CNT fiber showing the individual CNT bundles (nano-fibers) entangled into a nano-yarn with a nano-porous network structure. 


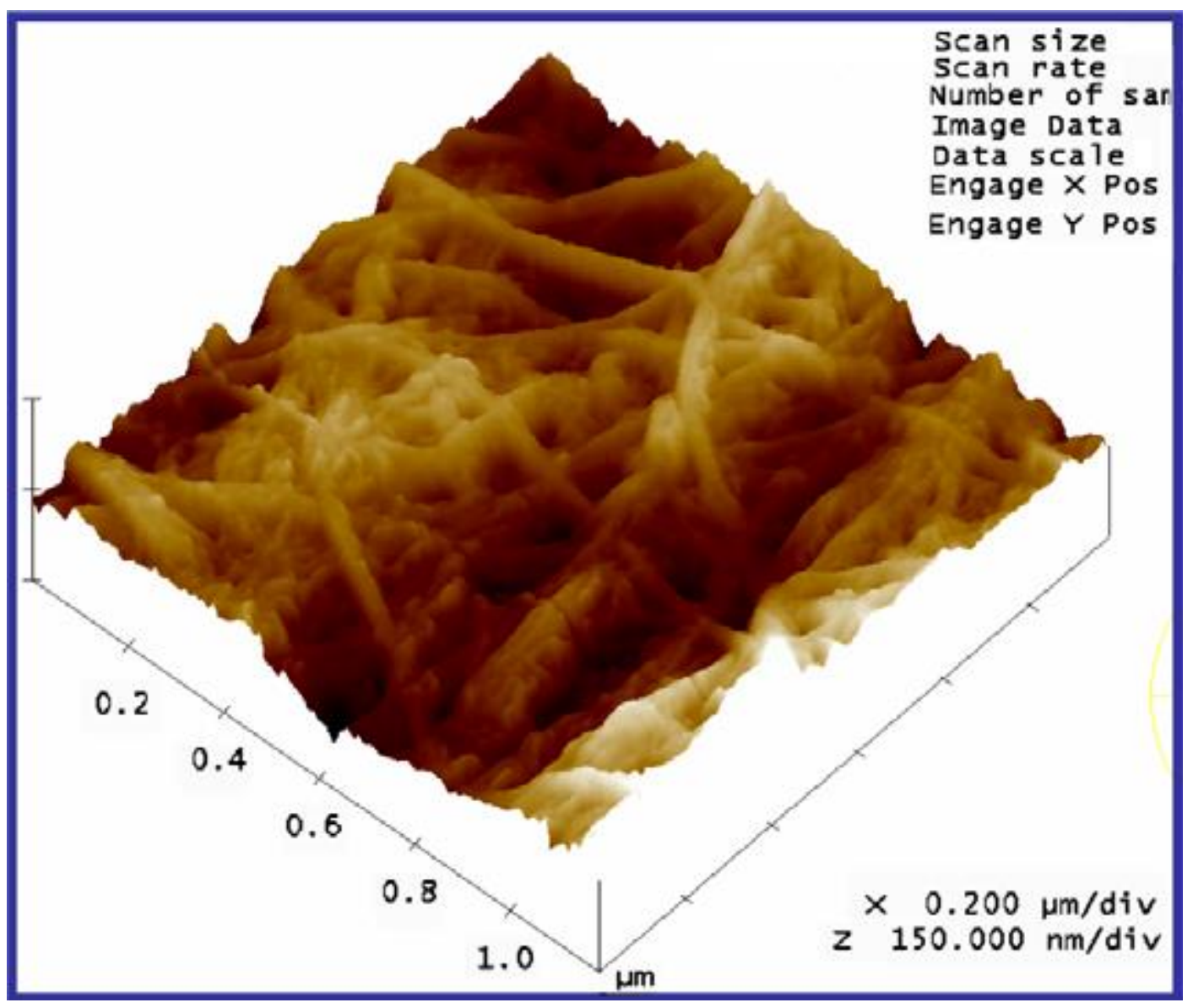

Figure 2. AFM photomicrograph showing the surface nano-topography of the CNT fiber. The nano-structure is consistent with the SEM photomicrograph, figure 1(b), showing the individual CNT bundles entangled into a nano-yarn with nano-porous network structure. 


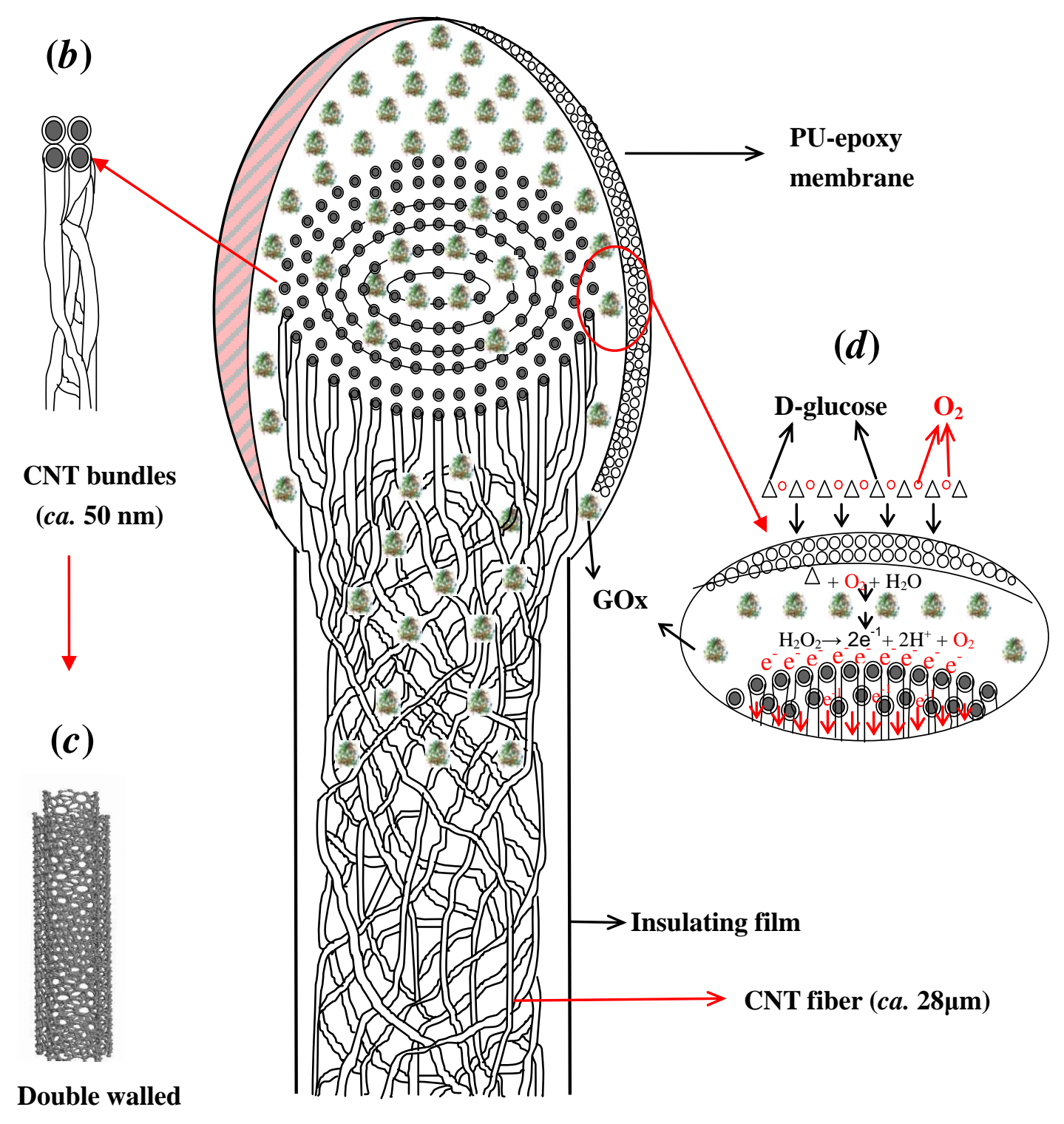

CNTs (ca. 8nm)

(a) CNT fiber glucose biosensor

Figure 3. Schematic diagram showing (a) CNT fiber based glucose biosensor, (b) CNT bundles, (c) DWNT and (d) working principle of biosensor. The CNT fiber (ca. $28 \mu \mathrm{m}$ ) is made of bundles (ca. $~ 50 \mathrm{~nm}$ ) of DWNTs (ca. $8-10 \mathrm{~nm}$ ) entangled to form concentrically compacted multiple layers of nano-yarns along the CNT fiber axis, as illustrated in (a) and in [19]. GOx enzyme is immobilized at the brush-like end of the CNT fiber and the enzyme layer is encapsulated by the epoxy-polyurethane (EPU) semi-permeable membrane. D-glucose and oxygen pass through the EPU membrane, GOx converts them into gluconic acid and hydro peroxide. The peroxide is converted to electrons, hydrogen and oxygen at a potential of $700 \mathrm{mV}$. The electrons are captured by the fiber, the signal from which is used to measure glucose concentrations. 

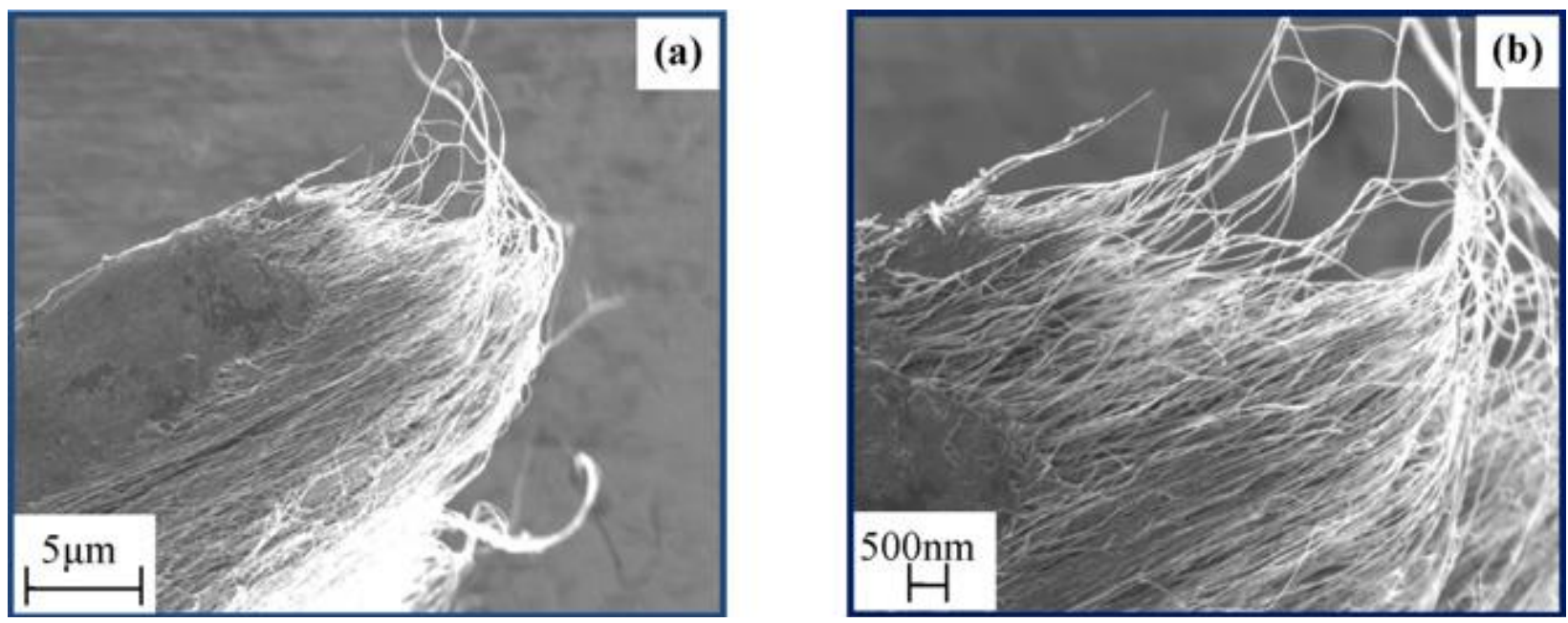

Figure 4. SEM photomicrograph showing the brush-like end of the CNT fiber prepared by snap freezing and twisting the end to unwind the nano-yarn. The separated individual CNT bundles are visible in the low (a) and high (b) magnification images. 


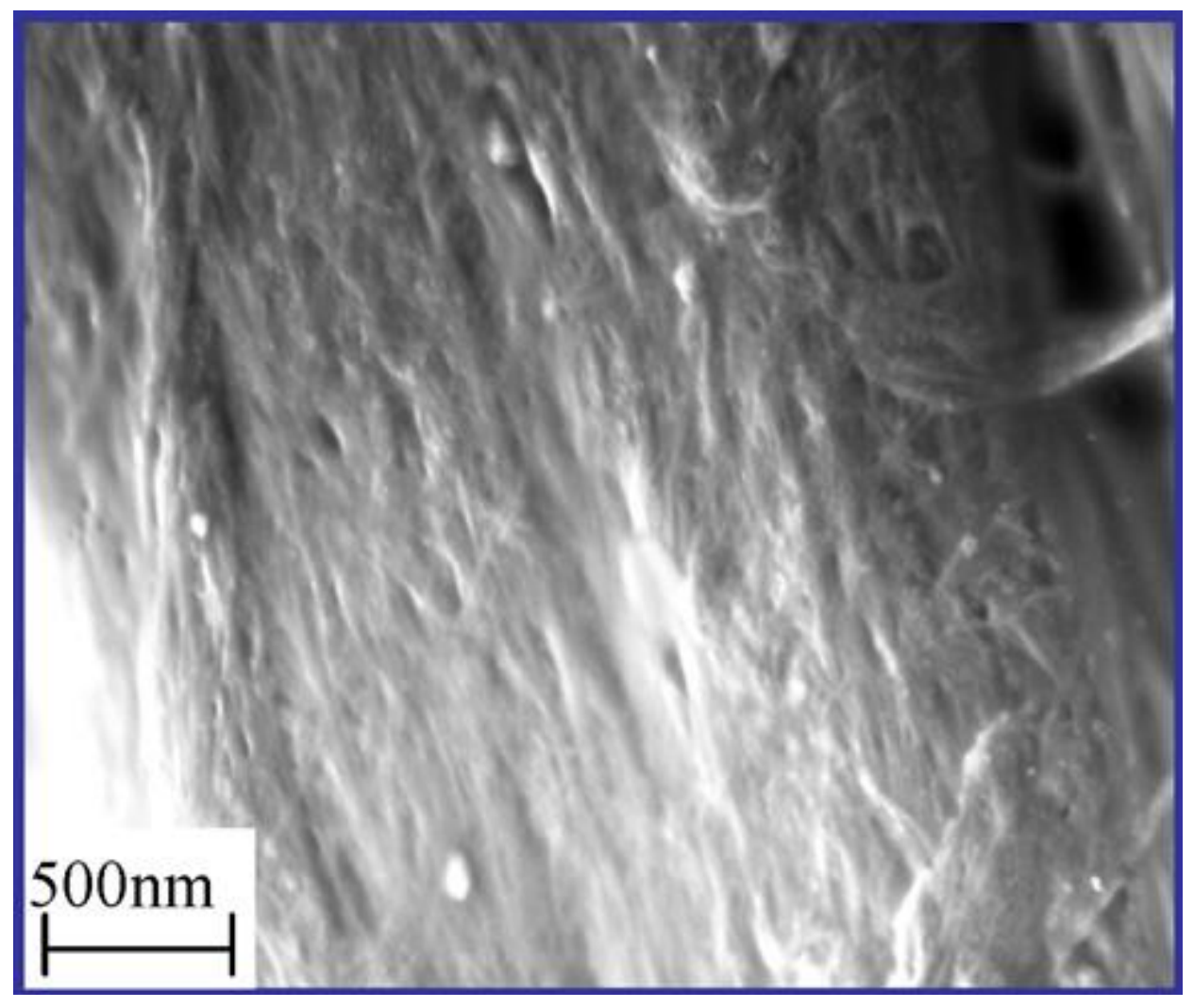

Figure 5. SEM photomicrograph showing the morphology of glucose oxidase enzyme immobilized on the CNT fiber. The enzyme layer appears to be uniform and snugly immobilized on the surface, evident from the contours showing the ridges and grooves corresponding to the individual CNT bundles and the nano-pores between them. 


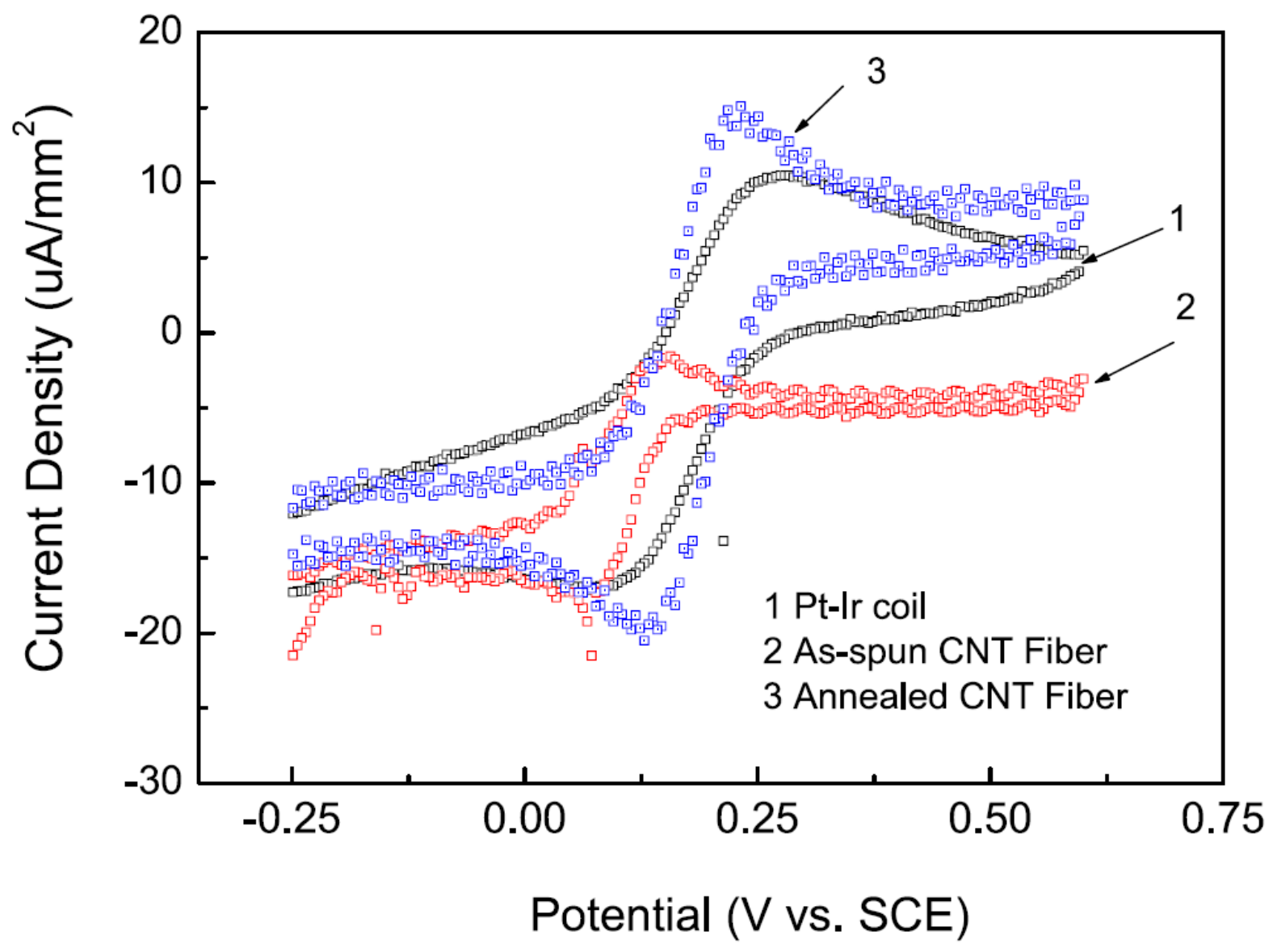

Figure 6. Cyclic voltammograms of different electrodes: (1) Pt-Ir coil, (2) as-spun CNT fiber, (3) annealed CNT fiber. The curves were obtained in a solution of $4 \mathrm{mM} \mathrm{K}_{3} \mathrm{Fe}(\mathrm{CN})_{6}$ in $0.1 \mathrm{mM} \mathrm{KCl}$ at a $50 \mathrm{mV} \mathrm{s}^{-1}$ scan rate. The anodic peak currents for Pt-Ir coil, as-spun and annealed CNT fiber electrodes are 11.3, 9.8 and $23.3 \mu \mathrm{A} \mathrm{mm}{ }^{-2}$ respectively; the cathodic peak currents are $15.2,13.7$ and $23.1 \mu \mathrm{A} \mathrm{mm}{ }^{-2}$ and potential differences between anodic and cathodic peaks $\left(\Delta E_{p}\right)$ are 177,90 and $93 \mathrm{eV}$, respectively. 


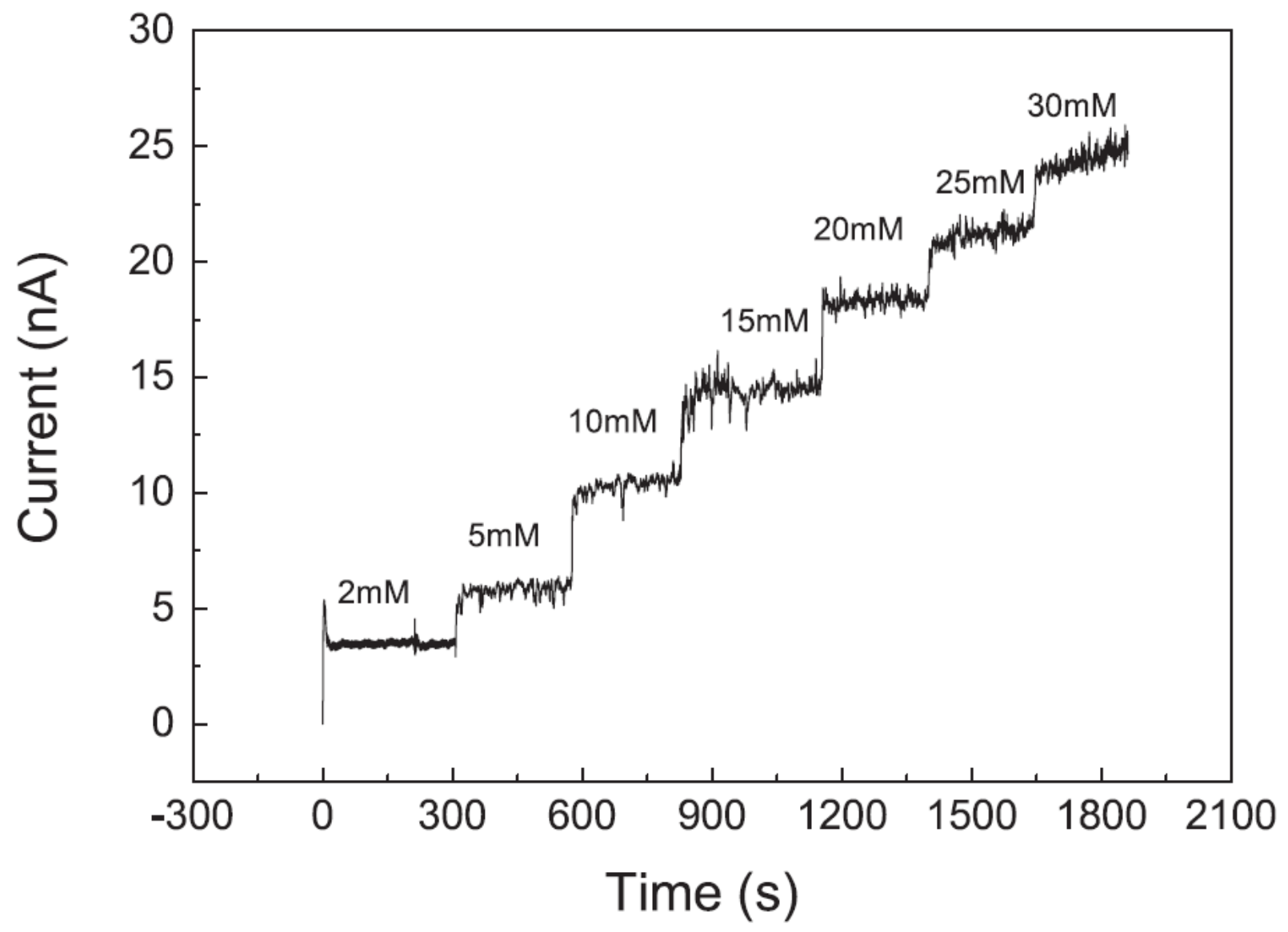

Figure 7. A typical amperometric response curve for a CNT fiber based glucose biosensor. Numbers in the chart represent the corresponding glucose concentration of the solution. The near instantaneous rise in the sensor response current following the addition of aliquots of $100 \mathrm{mM}$ glucose stock solution corresponding to 2, 5, 10, 15, 20, 25 and 30 $\mathrm{mM}$ final test concentrations are evident from the stepwise increase in the sensor response curve. 


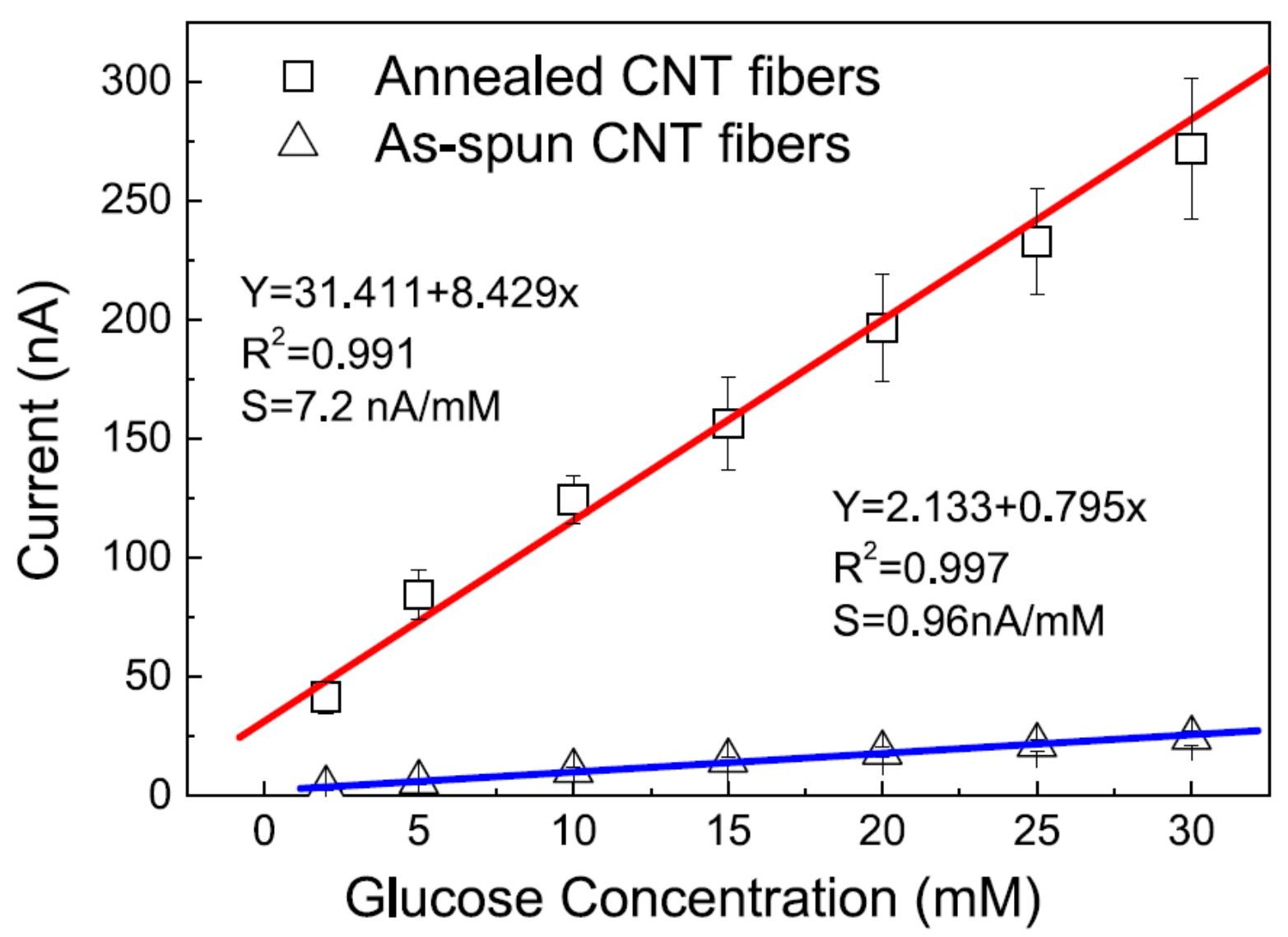

Figure 8. Calibration curves of the annealed and as-spun CNT fiber based glucose biosensors $(\mathrm{n}=3)$ showing linearity for the 2-30 $\mathrm{mM}$ glucose solution concentration range. 


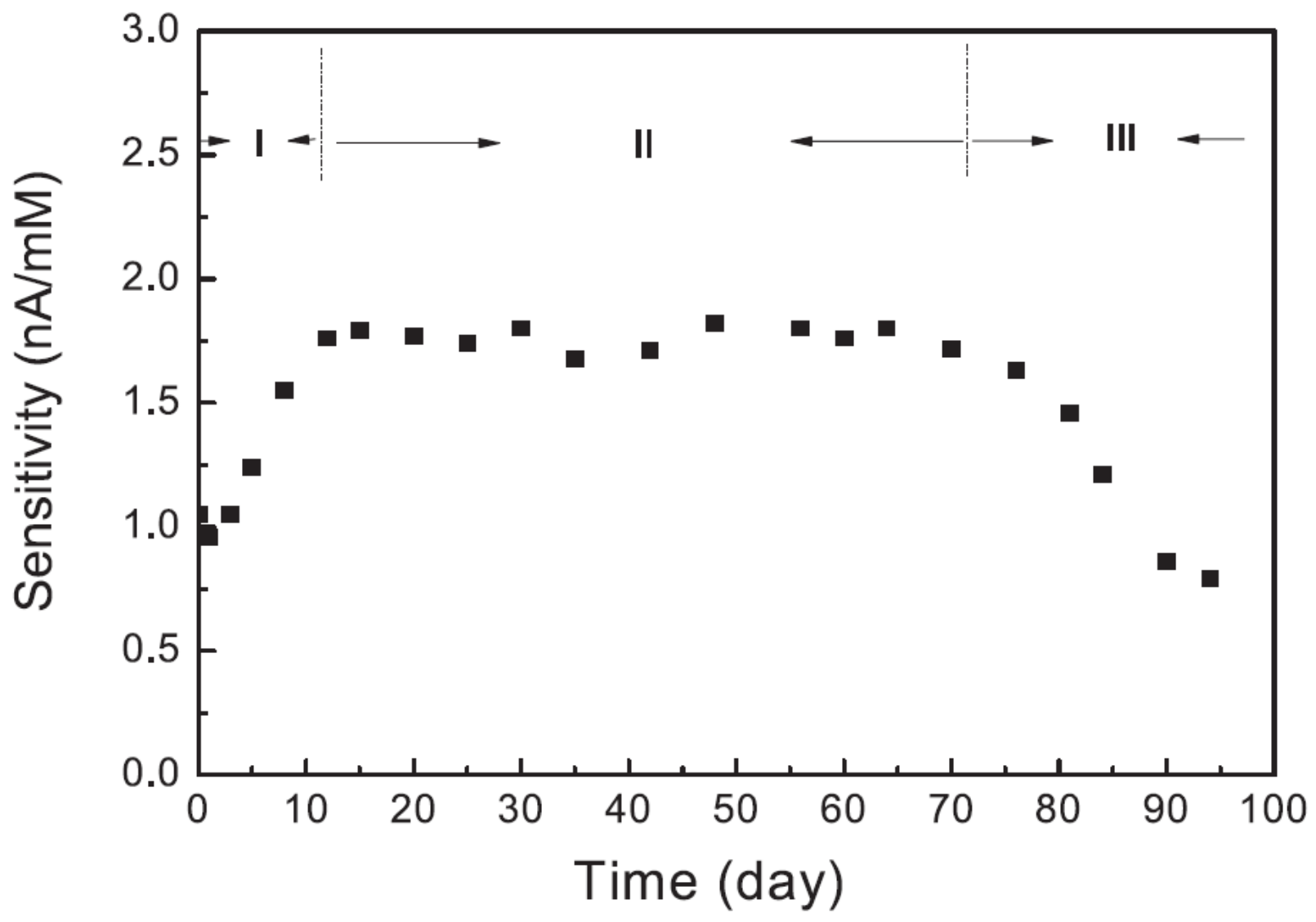

Figure 9. Long-term sensitivity variation of as-spun CNT fiber based glucose biosensors. Between the sensitivity testing, the sensors were stored in a $5 \mathrm{mM}$ glucose PBS solution at $24 \pm 1{ }^{\circ} \mathrm{C}$. 